\title{
Attitude Determination Methods Using Pseudolite Signal Phase Measurements
}

\author{
Keunjoo Park \\ Senior Researcher, Communication Satellite Systems Dept., COMS Program Office \\ Korea Aerospace Research Institute, Daejeon/South Korea \\ John L. Crassidis \\ Associate Professor, Dept. of Mechanical \& Aerospace Engineering \\ State University of New York, Amherst/NY
}

\begin{abstract}
In this paper, new attitude determination algorithms using pseudolite signal phase measurements are developed and presented with realistic simulations. Pseudolite signals are used to replace GPS signals which are often not available due to blocking by nearby large structures, for example a crew return vehicle under the International Space Station. A new observation model needs to be applied because spherical wavefronts are present for pseudolite signals, which are much closer to the antennas than GPS signals. Both static and filtering methods are developed. An iterative algorithm using the Levenberg-Marquardt method and a nonlinear Predictive filter provide a deterministic solution; however, they are not suitable for moving cases. Instead, the nonlinear Predictive filter, the extended Kalman filter and the Unscented filter using angular velocity measurements as well as pseudolite signal phase measurements are developed. In the convergence comparison, the Unscented filter and the Predictive filter show more robust behavior than that of the extended Kalman filter.
\end{abstract}

\section{Introduction}

The definition of attitude determination is to estimate the attitude parameters of a body fixed coordinate frame, relative to a reference coordinate frame, such as the Earth Centered Earth Fixed (ECEF) reference coordinate system. Due to the sensor type, attitude determination algorithms are largely divided into two approaches: static methods and filtering methods. Static methods provide a point-by-point attitude solution, while filtering methods combine dynamic and/or kinematic models. Both methods use measurements from attitude sensors, such as sun sensors, Earth sensors, a three-axis magnetometer (TAM), and/or star sensors. However, star sensors and sun sensors are generally very expensive. Also, they cannot be used in the case of eclipse or bright objects in sensor's field of view (FOV). Earth sensors cannot provide three-axis attitude information, and the estimation error using a TAM is relatively large.

The Global Positioning System (GPS) was originally developed for the purpose of navigation. With the pseudorange measurements of the GPS receiver the instantaneous positions and velocities are determined as well as the precise time. No other instrument can provide this information with both the accuracy and bandwidth achievable with GPS. In addition to orbit determination, the capability of GPS to provide attitude information makes it increasingly applied in the attitude subsystem of modern spacecraft [1-5]. Since GPS receivers can 
also measure the signal carrier phases, they have been applied as attitude sensors with multiple antenna sets. However, when using the phase measurements the solution becomes more complicated since the phase measurements contain integer ambiguities [6-8]. Furthermore, to utilize a GPS receiver as an attitude sensor, the system parameters such as baselines and line biases need to be determined, as well as integer ambiguities [4,9]. This operation is called the self survey. Once a self survey is accomplished, the attitude determination problem then can be solved.

In the key application by Cohen and Trimble Navigation, Ltd. in the late 1980's the GPS receivers, TANS Vector and TANS Quadrex, were designed primarily for airborne applications, tracking up to six satellites on four separate antennas [1]. Cohen has developed an iterative nonlinear least squares using Euler angles. When three non-coplanar baselines exist, Cohen showed that the solution based on Wahba's problem [10] is almost an order faster than a nonlinear least squares algorithm. Still, a singular value decomposition (SVD) that is computationally expensive must be performed. Bar-Itzhack et al. showed another analytical conversion of the GPS phase difference measurements into unit vectors to be used in QUEST algorithm [11]. However, it only used two baselines sets. Crassidis and Markley have developed a generalized deterministic attitude solution using GPS phase difference measurements $[12,13]$. Crassidis et al. have proposed an efficient and optimal algorithm based on nonlinear predictive filter scheme $[2,14]$. This algorithm, called Attitude-LeanLoping-Estimator using GPS Recursive Operations (ALLEGRO), has several advantages: 1) it is non-iterative, 2) an optimal attitude is provided even for coplanar baseline configurations, and 3) it guarantees convergence even for poor initial conditions [3].

Estimator-based filtering methods such as the extended Kalman filter (EKF) have also been developed for GPS attitude determination applications. The main advantage of using filtering techniques is that the three-axis attitude solution can be achieved using less than three baseline sets, as long as there is sufficient vehicle motion. Also, line biases can be estimated concurrently with the attitude. Fujikawa and Zimbelman developed an EKF using GPS signal phase differences to estimate the attitude and line biases using one baseline [15]. Crassidis et al. have proposed a new filter based on nonlinear predictive filter scheme [3]. This filter does not assume that the external torque is modeled by a zero-mean Gaussian process. Instead, it is determined during the estimation process. In the GPS receiver, data defined by a RINEX format the Signal-to-Noise Ratio (SNR) of GPS signal is also measured. Axelrad and Behre have developed an attitude determination algorithm using GPS SNR measurements [16]. Lightsey and Madsen developed an EKF algorithm using canted antenna SNR measurements [17]. However, the attitude errors of the SNR measurements methods are larger than those of using carrier phase measurements.

All of the various attitude determination approaches have been tested on a number of actual spacecraft [18-22]. Currently, the International Space Station (ISS) uses GPS for both orbit and attitude determination. However, significant GPS signal outages occur due to various structures near the ISS. Gaylor et al. showed that GPS signals below 10 meters from the ISS are blocked $99.99 \%$ [5]. Therefore, pseudolite techniques are being developing by the Navigation Systems and Technology Laboratory (NSTL) in NASA's Johnson Space Center (JSC) and Texas A\&M university to replace GPS signals near the proximity of the ISS [4]. The pseudolite transceivers are used to transmit GPS-like signals. However, this leads to more complicated solutions because the pseudolite signals have spherical wavefronts. These non-planar (or spherical) wavefronts effects were investigated for the rendezvous problem by Zimmerman $[23,24]$. In the attitude determination algorithms, these effects should be resolved. In this paper, optimal attitude determination algorithms using pseudolite signals are investigated. Levenberg-Marquardt method, Predictive filter, EKF, and Unscented filter (UF) [25] based algorithms are developed and analyzed with realistic simulations. 


\section{Problem Statement}

The main purpose of using pseudolite (PL) signals is to replace the GPS signals when the GPS signals may not be available [26,27]. For example, GPS signals are not available on the Moon or Mars. Also, they are often blocked by the nearby huge structures. Pseudolite signals are also used to determine relative attitude and positions [28,29]. Pseudolite signals are essentially the same as GPS signals except the signals are now being broadcasted by PL signal transceivers of which positions are known. When the PL signals are used, the phase measurement model should be modified because the PL signal transceiver (TX) is located too close to antennas so that the planar assumption of GPS signals does not hold. Since the relative distances between antennas and PL TXs are short, relative position vectors between them are no longer assumed as parallel. Instead, a spherical wavefront model was applied $[23,24,30]$. In Figure 1 the PL signal phase measurement model is shown. As can

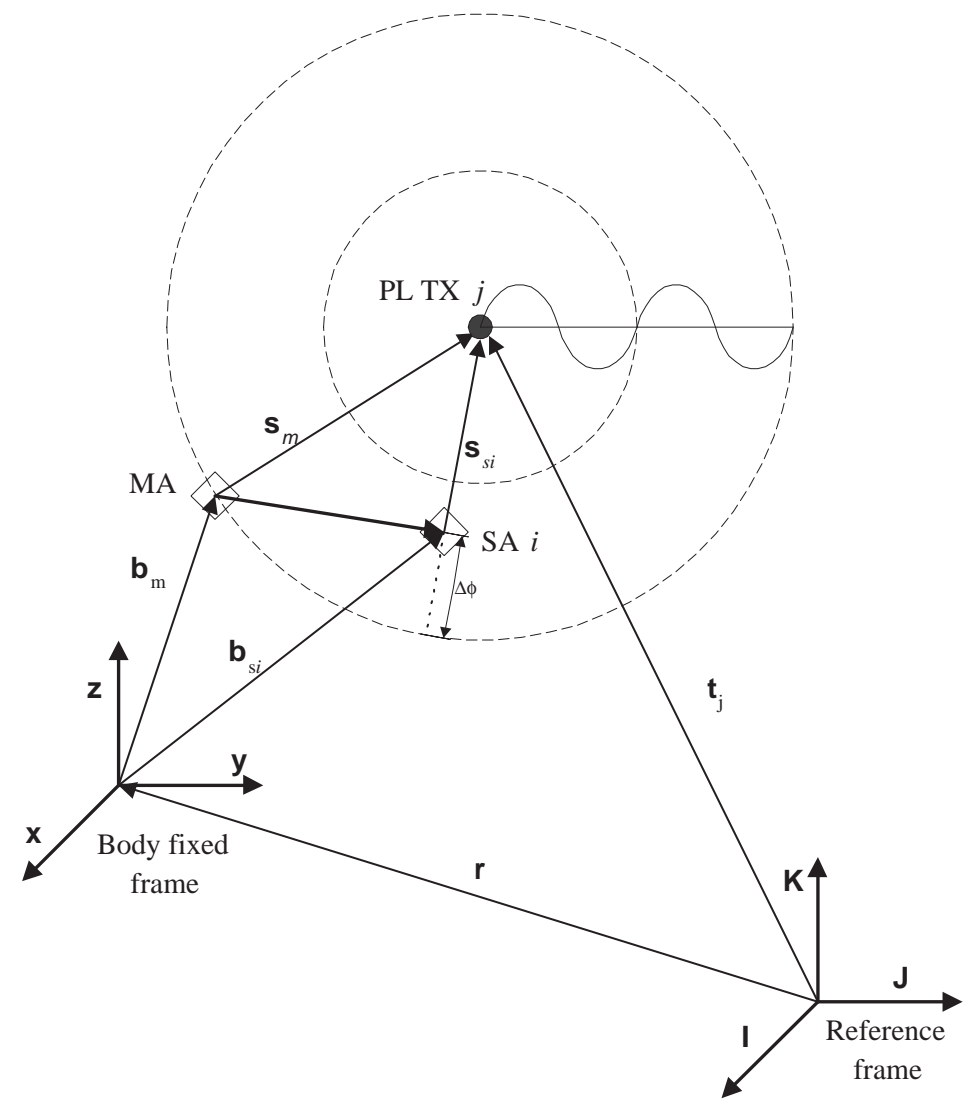

Figure 1: Non-Planar Pseudolite Carrier Phase Measurement Model

be seen, the phase measurement contains the nonlinear spherical wavefront effect. The PL signal phase measurement can be expressed by

$$
\Delta \phi_{i j}=\left(\left|\mathbf{r}+A^{\top} \mathbf{b}_{m}-\mathbf{t}_{j}\right|-\left|\mathbf{r}+A^{\top} \mathbf{b}_{s_{i}}-\mathbf{t}_{j}\right|\right) / \lambda+n_{i j}+\tau_{i}
$$

where $A$ is the attitude matrix, which transforms coordinates from the reference frame to body frame, $n$ is the integer ambiguity, $\tau$ is the line bias error, $\lambda$ is the wavelength, $\mathbf{t}_{j}$ is the position vector of the $j$-th PL TX in the reference frame, $\mathbf{r}$ is the position vector of the body frame origin in the reference frame, $\mathbf{b}_{m}$ is the position vector of the master antenna (MA) in the body frame, $\mathbf{b}_{s_{i}}$ is the position vector of the $i$-th slave antenna (SA) in the body fixed frame. 
It is assumed that the baselines, line biases, and integer ambiguities are already determined using a self survey process. Therefore, the attitude determination is a nonlinear estimation problem to estimate the attitude parameters.

\section{Previous Work}

The most widely used attitude determination techniques using LOS vector measurements are methods to solve Wahba's problem [10]. Although the GPS signal phase measurements are not LOS vectors, a modified Wahba's problem was posed by Crassidis et al. [11-13]. They also found a suboptimal attitude solution. Nonlinear least squares or gradient-based search techniques can solve for the optimal attitude by minimizing a certain loss function. However, these methods are not computationally efficient because they are iterative. Also, convergence is not guaranteed if the initial errors are large. A non-iterative algorithm using predictive filtering scheme, called ALLEGRO, was developed by Crassidis [3]. Fujikawa and Zimbelman developed an EKF using GPS signal phase measurements to estimate the attitude and line biases using one baseline [15].

\section{New Approach}

Since the signals are no longer planar, new attitude determination algorithms using the PL signal phase measurement model need to be developed. In this paper, both batch and filtering methods are developed. As for batch methods, the Levenberg-Marquardt (LM) algorithm and the modified ALLEGRO are developed. For the filtering methods which use angular velocity measurements as well as PL signal phase measurements, the EKF and the UF are developed.

\section{Attitude Determination Methods Using PL Signals}

\section{Levenberg-Marquardt Method}

A Levenberg-Marquardt method using the quaternion is developed. Using the quaternion [31], the estimated attitude matrix can be written by

$$
A(\hat{\mathbf{q}})=A(\delta \mathbf{q}) A(\mathbf{q})
$$

where $\hat{\mathbf{q}}$ represented the estimated quaternion, $\delta \mathbf{q}$ is the error quaternion, and $\mathbf{q}$ is the true quaternion. In the small angle approximation, $A(\delta \mathbf{q})$ can be rewritten as

$$
A(\delta \mathbf{q})=\mathbf{I}-2\left[\delta \mathbf{q}_{13} \times\right]
$$

where $\delta \mathbf{q}_{13}$ is the vector part of the error quaternion and

$$
\left[\delta \mathbf{q}_{13} \times\right]=\left[\begin{array}{rrr}
0 & -\delta q_{3} & \delta q_{2} \\
\delta q_{3} & 0 & -\delta q_{1} \\
-\delta q_{2} & \delta q_{1} & 0
\end{array}\right]
$$

Then, the PL signal phase measurement model in Eq. (1) can be approximated as

$$
\begin{aligned}
\Delta \hat{\phi}_{i j} \approx & {\left[\sqrt{\ell_{m}}-2\left(\mathbf{r}+A^{\top} \mathbf{b}_{m}-\mathbf{t}_{j}\right)^{\top} A^{\top}\left[\mathbf{b}_{m} \times\right] \delta \mathbf{q} / \sqrt{\ell_{m}}\right.} \\
& \left.-\sqrt{\ell_{s_{i}}}-2\left(\mathbf{r}+A^{\top} \mathbf{b}_{s_{i}}-\mathbf{t}_{j}\right)^{\top} A^{\top}\left[\mathbf{b}_{s_{i}} \times\right] \delta \mathbf{q} / \sqrt{\ell_{s_{i}}}\right] / \lambda+n_{i j}+\tau_{i}
\end{aligned}
$$

where

$$
\begin{aligned}
\ell_{m} & =\left(\mathbf{r}+A^{\top} \mathbf{b}_{m}-\mathbf{t}_{j}\right)^{\top}\left(\mathbf{r}+A^{\top} \mathbf{b}_{m}-\mathbf{t}_{j}\right) \\
\ell_{s_{i}} & =\left(\mathbf{r}+A^{\top} \mathbf{b}_{s_{i}}-\mathbf{t}_{j}\right)^{\top}\left(\mathbf{r}+A^{\top} \mathbf{b}_{s_{i}}-\mathbf{t}_{j}\right)
\end{aligned}
$$


Thus, the Jacobian matrix is given by

$$
\begin{aligned}
\mathbf{H}_{i j} & =\frac{\partial \Delta \hat{\phi}_{i j}}{\partial \delta \mathbf{q}_{13}} \\
& \approx-\frac{2}{\lambda}\left\{\frac{\left(\mathbf{r}+A^{\top} \mathbf{b}_{m}-\mathbf{t}_{j}\right)^{\top} A^{\top}\left[\mathbf{b}_{m} \times\right]}{\sqrt{\ell_{m}}}-\frac{\left(\mathbf{r}+A^{\top} \mathbf{b}_{s_{i}}-\mathbf{t}_{j}\right)^{\top} A^{\top}\left[\mathbf{b}_{s_{i}} \times\right]}{\sqrt{\ell_{s_{i}}}}\right\}
\end{aligned}
$$

To compute the attitude covariance matrix, the Fisher information matrix is determined by

$$
\begin{aligned}
\mathbf{F} & \equiv \frac{1}{4} E\left\{\frac{\partial J}{\partial \mathbf{q}_{13}}{\frac{\partial J}{\partial \mathbf{q}_{13}}}^{\top}\right\} \\
& =\frac{1}{4} \sum_{i=1}^{m} \sum_{j=1}^{n} \sigma_{i j}^{-2} \mathbf{H}_{i j}^{\top} \mathbf{H}_{i j}
\end{aligned}
$$

where the division by a factor of 4 is required because quaternion errors are two times the Euler angle errors and the loss function is defined by

$$
J=\frac{1}{2} \sum_{i=1}^{m} \sum_{j=1}^{n} \sigma_{i j}^{-2}\left(\Delta \tilde{\phi}_{i j}-\Delta \phi_{i j}\right)^{2}
$$

where $m$ represents the number of baselines, $n$ represents the number of sightlines, and $\sigma_{i j}$ denotes the standard deviation of the $i j$-th measurement error. Then, the attitude error covariance matrix is obtained by

$$
\begin{aligned}
\mathbf{P} & \equiv \mathbf{F}^{-1} \\
& =4\left[\sum_{i=1}^{m} \sum_{j=1}^{n} \sigma_{i j}^{-2} \mathbf{H}_{i j}^{\top} \mathbf{H}_{i j}\right]^{-1}
\end{aligned}
$$

For large initial guess errors, nonlinear least squares using the Jacobian matrix in Eq. (7) may not converge to correct solutions. The method of steepest descent many help to avoid this problem, however, the convergence is very poor close to the solution [32]. These difficulties can be overcome by LM method [33]. In the LM method, the search direction is intermediate between the steepest descent and the differential correction direction such as nonlinear least squares, which makes the convergence more robust.

\section{Nonlinear Predictive Filter}

An algorithm using the nonlinear Predictive filter from GPS signals was proposed by Crassidis et al. $[2,3,14]$. The major advantage of the Predictive filter is that the model error is not assumed to be represented by a zero-mean Gaussian noise process with known covariance, but instead is determined during the estimation process [34]. Crassidis et al. have proposed the nonlinear Predictive filter by simultaneously solving system optimality conditions and an output error constraint. Since the GPS signal multipath error is known to have non-Gaussian components, this approach is fit for PL signal application because the multipath effect of PL signal is more severe than that of GPS signal. This algorithm is called the Attitude Lean Loping Estimator using GPS Recursive Operations (ALLEGRO). In the ALLEGRO algorithm, the model is assumed as the quaternion kinematics model. Also, the attitude rate is adequately modeled by a constant model error $\mathbf{d}$ between measurements, which is given by

$$
\dot{\hat{\mathbf{q}}}=\frac{1}{2} \boldsymbol{\Xi}(\hat{\mathbf{q}}) \mathbf{d}
$$

where $\hat{\mathbf{q}}$ denotes the estimated quaternion. Therefore, the attitude parameter, i.e. the quaternion, is determined without using angular rate information. The loss function to be minimized is given by

$$
J[\mathbf{d}(t)]=\frac{1}{2}[\tilde{\boldsymbol{y}}(t+\Delta t)-\hat{\boldsymbol{y}}(t+\Delta t)]^{\top} \mathbf{R}^{-1}[\tilde{\boldsymbol{y}}(t+\Delta t)-\hat{\boldsymbol{y}}(t+\Delta t)]+\frac{1}{2} \mathbf{d}^{\top}(t) \mathbf{W} \mathbf{d}(t)
$$


where $\Delta t$ is the measurement sampling interval, $\mathbf{W} \in \mathrm{R}^{q \times q}$ is the positive semi-definite weighting matrix, $\tilde{\boldsymbol{y}}(t+\Delta t)$ is the measurement at time $t+\Delta t$, and $\hat{\boldsymbol{y}}(t+\Delta t)$ is the estimated output at time $t+\Delta t$. The optimal model error can be obtained by minimizing Eq. (12) with respect to $\mathbf{d}(t)$, given by

$$
\begin{gathered}
\mathbf{d}(t)=-\left\{[\boldsymbol{\Lambda}(\Delta t) \mathbf{S}(\hat{\boldsymbol{x}})]^{\top} \mathbf{R}^{-1} \boldsymbol{\Lambda}(\Delta t) \mathbf{S}(\hat{\boldsymbol{x}})+\mathbf{W}\right\}^{-1}[\boldsymbol{\Lambda}(\Delta t) \mathbf{S}(\hat{\boldsymbol{x}})]^{\top} \mathbf{R}^{-1} \\
{[\boldsymbol{z}(\hat{\mathbf{x}}, \Delta t)-\tilde{\boldsymbol{y}}(t+\Delta t)+\hat{\boldsymbol{y}}(t)]}
\end{gathered}
$$

where the matrix $\mathbf{S}[\hat{\mathbf{q}}(t)]$ is a generalized sensitivity matrix, and $\boldsymbol{\Lambda}(\Delta t) \in \mathrm{R}^{m \times m}$ is a diagonal matrix with elements given by

$$
\lambda_{i i}=\Delta t
$$

The $i$-th component of vector $\boldsymbol{z}[\hat{\mathbf{q}}(t), t]$ is given by

$$
z_{i}(\hat{\mathbf{q}}, \Delta t)=\Delta t L_{f}^{1}\left(c_{i}\right)
$$

where $L_{f}^{1}\left(c_{i}\right)$ is the 1-st Lie derivative and $\mathbf{c}[\hat{\mathbf{q}}(t), t] \in \mathrm{R}^{n} \rightarrow \mathrm{R}^{m}$ is the output vector. Then, the obtained $\mathbf{d}(t)$ in Eq. (13) is used to perform a nonlinear propagation of the state estimates in Eq. (11) to time $t$. So, the measurement is processed at time $t+\Delta t$ to find the new $\mathbf{d}(t)$ in $[t, t+\Delta t]$, and then the state estimates are propagated to time $t+\Delta t$. As $\mathbf{W}$ decreases, more model error is added to correct the model, so that the estimates more closely follow the measurements. As $\mathbf{W}$ increases, less model error is added, so that the estimates more closely follow the propagated model.

A modified ALLEGRO using the PL signal observation model in Eq. (1) is now developed. To derive the $\mathbf{S}(\hat{\mathbf{q}})$ matrix , the following matrices are defined

$$
\boldsymbol{\Omega}(\mathbf{a}) \equiv\left[\begin{array}{ccc}
-[\mathbf{a} \times] & \vdots & -\mathbf{a} \\
\ldots \ldots \ldots & \ldots . . . \\
-\mathbf{a}^{\top} & \vdots & 0
\end{array}\right], \quad \boldsymbol{\Gamma}(\mathbf{b}) \equiv\left[\begin{array}{ccc}
-[\mathbf{b} \times] & \vdots & -\mathbf{b} \\
\ldots \ldots \ldots \ldots \ldots . \\
\mathbf{b}^{\top} & \vdots & 0
\end{array}\right]
$$

where $\mathbf{a}$ and $\mathbf{b}$ are 3 -dimensional vectors. Another useful properties between quaternion $\mathbf{q}$ and 3 -dimensional vectors are given by

$$
\begin{aligned}
& \boldsymbol{\Omega}(\mathbf{a}) \mathbf{q}=\boldsymbol{\Xi}(\mathbf{q}) \mathbf{a} \\
& \Gamma(\mathbf{b}) \mathbf{q}=\boldsymbol{\Psi}(\mathbf{q}) \mathbf{b}
\end{aligned}
$$

where matrices $\boldsymbol{\Xi}(\mathbf{q})$ and $\boldsymbol{\Psi}(\mathbf{q})$ are defined by

$$
\boldsymbol{\Xi}(\mathbf{q}) \equiv\left[\begin{array}{c}
q_{4} \mathbf{I}_{3 \times 3}+\left[\mathbf{q}_{13} \times\right] \\
\ldots \ldots \ldots \ldots \ldots \\
-\mathbf{q}_{13}^{\top}
\end{array}\right], \quad \boldsymbol{\Psi}(\mathbf{q}) \equiv\left[\begin{array}{c}
q_{4} \mathbf{I}_{3 \times 3}-\left[\mathbf{q}_{13} \times\right] \\
\ldots \ldots \ldots \ldots \ldots \\
-\mathbf{q}_{13}^{\top}
\end{array}\right]
$$

Using the property $\mathbf{A}(\mathbf{q})=\boldsymbol{\Xi}^{\top}(\mathbf{q}) \boldsymbol{\Psi}(\mathbf{q})$ and substituting Eqs. (16), (17), and (18) into Eq. (1) yields

$$
\begin{aligned}
c_{i j}[\hat{\mathbf{q}}(t), t] & =\frac{\sqrt{\left(\mathbf{r}+A^{\top}(\hat{\mathbf{q}}) \mathbf{b}_{m}-\mathbf{t}_{j}\right)^{\top}\left(\mathbf{r}+\mathbf{A}^{\top}(\hat{\mathbf{q}}) \mathbf{b}_{m}-\mathbf{t}_{j}\right)}-\sqrt{\left(\mathbf{r}+A^{\top} \mathbf{b}_{s_{i}}-\mathbf{t}_{j}\right)^{\top}\left(\mathbf{r}+A^{\top}(\hat{\mathbf{q}}) \mathbf{b}_{s_{i}}-\mathbf{t}_{j}\right)}}{\lambda} \\
& =\frac{\sqrt{\ell+\mathbf{b}_{m}^{\top} \mathbf{b}_{m}-2 \hat{\mathbf{q}}^{\top} \boldsymbol{\Omega}\left(\mathbf{b}_{m}\right) \boldsymbol{\Gamma}\left(\mathbf{t}_{j}-\mathbf{r}\right) \hat{\mathbf{q}}}-\sqrt{\ell+\mathbf{b}_{s_{i}}^{\top} \mathbf{b}_{s_{i}}-2 \hat{\mathbf{q}}^{\top} \boldsymbol{\Omega}\left(\mathbf{b}_{s_{i}}\right) \boldsymbol{\Gamma}\left(\mathbf{t}_{j}-\mathbf{r}\right) \hat{\mathbf{q}}}}{\lambda}
\end{aligned}
$$

where $\ell=\mathbf{r}^{\top} \mathbf{r}-2 \mathbf{r}^{\top} \mathbf{t}_{j}+\mathbf{t}_{j}^{\top} \mathbf{t}_{j}$. The matrix $\mathbf{S}(\hat{\mathbf{q}})$ is formed by taking the partial derivative of Eq. (19) with respect to $\hat{\mathbf{q}}$ and right-multiplying by $\boldsymbol{\Xi}(\hat{\mathbf{q}}) / 2$. Therefore, we have

$$
\mathbf{S}(\hat{\mathbf{q}})=\frac{1}{2 \lambda}\left[\begin{array}{c}
\frac{\hat{\mathbf{q}}^{\top}\left[\boldsymbol{\Omega}\left(\mathbf{b}_{s_{1}}\right) \boldsymbol{\Gamma}\left(\mathbf{t}_{1}-\mathbf{r}\right)+\boldsymbol{\Gamma}\left(\mathbf{t}_{1}-\mathbf{r}\right) \boldsymbol{\Omega}\left(\mathbf{b}_{s_{1}}\right)\right]}{\sqrt{\ell+\mathbf{b}_{s_{1}}^{\top} \mathbf{b}_{s_{1}}-2 \hat{\mathbf{q}}^{\top} \boldsymbol{\Omega}\left(\mathbf{b}_{s_{1}}\right) \boldsymbol{\Gamma}\left(\mathbf{t}_{1}-\mathbf{r}\right) \hat{\mathbf{q}}}}-\frac{\hat{\mathbf{q}}^{\top}\left[\boldsymbol{\Omega}\left(\mathbf{b}_{m}\right) \boldsymbol{\Gamma}\left(\mathbf{t}_{1}-\mathbf{r}\right)+\boldsymbol{\Gamma}\left(\mathbf{t}_{1}-\mathbf{r}\right) \boldsymbol{\Omega}\left(\mathbf{b}_{m}\right)\right]}{\sqrt{\ell+\mathbf{b}_{m}^{\top} \mathbf{b}_{m}-2 \hat{\mathbf{q}}^{\top} \boldsymbol{\Omega}\left(\mathbf{b}_{m}\right) \boldsymbol{\Gamma}\left(\mathbf{t}_{1}-\mathbf{r}\right) \hat{\mathbf{q}}}} \\
\vdots \\
\frac{\hat{\mathbf{q}}^{\top}\left[\boldsymbol{\Omega}\left(\mathbf{b}_{s_{m}}\right) \boldsymbol{\Gamma}\left(\mathbf{t}_{n}-\mathbf{r}\right)+\boldsymbol{\Gamma}\left(\mathbf{t}_{n}-\mathbf{r}\right) \boldsymbol{\Omega}\left(\mathbf{b}_{s_{m}}\right)\right]}{\sqrt{\ell+\mathbf{b}_{s_{m}}^{\top} \mathbf{b}_{s_{m}}-2 \hat{\mathbf{q}}^{\top} \boldsymbol{\Omega}\left(\mathbf{b}_{s_{m}}\right) \boldsymbol{\Gamma}\left(\mathbf{t}_{n}-\mathbf{r}\right) \hat{\mathbf{q}}}}-\frac{\hat{\mathbf{q}}^{\top}\left[\boldsymbol{\Omega}\left(\mathbf{b}_{m}\right) \boldsymbol{\Gamma}\left(\mathbf{t}_{n}-\mathbf{r}\right)+\boldsymbol{\Gamma}\left(\mathbf{t}_{n}-\mathbf{r}\right) \boldsymbol{\Omega}\left(\mathbf{b}_{m}\right)\right]}{\sqrt{\ell+\mathbf{b}_{m}^{\top} \mathbf{b}_{m}-2 \hat{\mathbf{q}}^{\top} \boldsymbol{\Omega}\left(\mathbf{b}_{m}\right) \boldsymbol{\Gamma}\left(\mathbf{t}_{n}-\mathbf{r}\right) \hat{\mathbf{q}}}}
\end{array}\right] \boldsymbol{\Xi}(\hat{\mathbf{q}})
$$


The remaining quantities in Eq. (13) are given by

$$
\hat{\mathbf{y}}=\left[\begin{array}{c}
\boldsymbol{\Lambda}=\Delta t \mathbf{I}_{m \times m} \\
\frac{\sqrt{\ell+\mathbf{b}_{m}^{\top} \mathbf{b}_{m}-2 \hat{\mathbf{q}}^{\top} \boldsymbol{\Omega}\left(\mathbf{b}_{m}\right) \boldsymbol{\Gamma}\left(\mathbf{t}_{1}-\mathbf{r}\right) \hat{\mathbf{q}}}-\sqrt{\ell+\mathbf{b}_{s_{1}}^{\top} \mathbf{b}_{s_{1}}-2 \hat{\mathbf{q}}^{\top} \boldsymbol{\Omega}\left(\mathbf{b}_{s_{1}}\right) \boldsymbol{\Gamma}\left(\mathbf{t}_{1}-\mathbf{r}\right) \hat{\mathbf{q}}}}{\lambda} \\
\vdots \\
\frac{\sqrt{\ell+\mathbf{b}_{m}^{\top} \mathbf{b}_{m}-2 \hat{\mathbf{q}}^{\top} \boldsymbol{\Omega}\left(\mathbf{b}_{m}\right) \boldsymbol{\Gamma}\left(\mathbf{t}_{n}-\mathbf{r}\right) \hat{\mathbf{q}}}-\sqrt{\ell+\mathbf{b}_{s_{m}}^{\top} \mathbf{b}_{s_{m}}-2 \hat{\mathbf{q}}^{\top} \boldsymbol{\Omega}\left(\mathbf{b}_{s_{m}}\right) \boldsymbol{\Gamma}\left(\mathbf{t}_{n}-\mathbf{r}\right) \hat{\mathbf{q}}}}{\lambda} \\
\boldsymbol{z}(\hat{\boldsymbol{x}}, \Delta t)=\mathbf{0}
\end{array}\right]
$$

As shown in Ref. [3], the attitude error covariance using Eqs. (11) and (13) leads to the same covariance of Eq. (10) if $\Delta t$ is small. Therefore, the predictive approach leads to an efficient estimator.

\section{Extended Kalman Filter}

In this section, a quaternion based extended Kalman filter (EKF) using the PL signal phase measurement with gyro measurements is developed for attitude estimation. The state error vector has seven components consisting of error quaternion $\delta \mathbf{q}$ and gyro bias error $\Delta \boldsymbol{\beta}$. The multiplicative error quaternion is defined by [31]

$$
\delta \mathbf{q}=\mathbf{q} \otimes \hat{\mathbf{q}}^{-1}
$$

where $\mathbf{q}$ is the true quaternion, $\hat{\mathbf{q}}$ is the estimated quaternion, and the operator $\otimes$ refers to quaternion multiplication. The inverse quaternion is given by $\mathbf{q}^{-1}=\left[-\mathbf{q}_{13}^{\top} q_{4}\right]^{\top}$. However, the covariance matrix of the error quaternion is nearly singular since it has four components. Lefferts et al. solved this problem by reducing the covariance into a three-component representation [35]. The dimension of the covariance matrix is then 6 by 6 . For a small rotation, the error quaternion in Eq. (22) can be approximated by

$$
\delta \mathbf{q} \approx\left[\begin{array}{c}
\delta \mathbf{q}_{13} \\
1
\end{array}\right]
$$

The vector part of the error quaternion, $\delta \mathbf{q}_{13}$, corresponds to half Euler angle errors for a small angle approximation. By using the quaternion kinematics model and the typical gyro model, the state model equation can be written by

$$
\begin{gathered}
\dot{\mathbf{q}}=\frac{1}{2} \boldsymbol{\Omega}\left(\widetilde{\boldsymbol{\omega}}-\boldsymbol{\beta}-\boldsymbol{\eta}_{v}\right) \mathbf{q} \\
\dot{\boldsymbol{\beta}}=\boldsymbol{\eta}_{u}
\end{gathered}
$$

where $\boldsymbol{\eta}_{v}$ and $\boldsymbol{\eta}_{u}$ are zero-mean Gaussian white-noise processes with the properties:

$$
\begin{gathered}
E\left[\boldsymbol{\eta}_{v}(s) \boldsymbol{\eta}_{v}(\tau)\right]=\sigma_{v}^{2} \delta(s-\tau) \mathbf{I}_{3 \times 3} \\
E\left[\boldsymbol{\eta}_{u}(s) \boldsymbol{\eta}_{u}(\tau)\right]=\sigma_{u}^{2} \delta(s-\tau) \mathbf{I}_{3 \times 3} \\
E\left[\boldsymbol{\eta}_{v}(s) \boldsymbol{\eta}_{u}(\tau)\right]=\mathbf{0}_{3 \times 3}
\end{gathered}
$$

where $\delta(t)$ is the Dirac delta function. By using the vector part of the error quaternion in Eq. (23) and the additive gyro drift error $\Delta \boldsymbol{\beta}$, the state error equation of the EKF is written as

$$
\Delta \dot{x}=\mathbf{f}_{x} \Delta x+\mathbf{g}_{x} w
$$

where the state error is given by

$$
\Delta \boldsymbol{x}=\left[\begin{array}{c}
\delta \mathbf{q}_{13} \\
\Delta \boldsymbol{\beta}
\end{array}\right]
$$


The Jacobian matrices are given by

$$
\begin{gathered}
\mathbf{f}_{\boldsymbol{x}}=\left[\begin{array}{cc}
-[\widehat{\boldsymbol{\omega}} \times] & -\frac{1}{2} \mathbf{I}_{3 \times 3} \\
\mathbf{0}_{3 \times 3} & \mathbf{0}_{3 \times 3}
\end{array}\right] \\
\mathbf{g}_{\boldsymbol{x}}=\left[\begin{array}{cc}
-\frac{1}{2} \mathbf{I}_{3 \times 3} & \mathbf{0}_{3 \times 3} \\
\mathbf{0}_{3 \times 3} & \mathbf{I}_{3 \times 3}
\end{array}\right]
\end{gathered}
$$

where the angular velocity estimate is given by

$$
\widehat{\boldsymbol{\omega}}=\widetilde{\boldsymbol{\omega}}-\widehat{\boldsymbol{\beta}}
$$

For the state-observable discrete measurements model of the PL signal shown in Eq. (1), the corresponding $\mathbf{H}_{k}$ matrix is given by

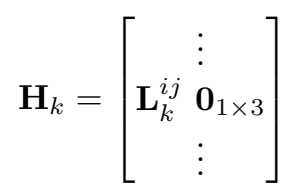

where

$$
\begin{aligned}
\mathbf{L}_{k}^{i j} & =\frac{\partial \Delta \phi_{i j}}{\partial \delta \mathbf{q}_{13}} \\
& \approx-\frac{2}{\lambda}\left\{\frac{\left(\mathbf{r}+A^{\top}\left(\hat{\mathbf{q}}_{k}^{-}\right) \mathbf{b}_{m}-\mathbf{t}_{j}\right)^{\top} A^{\top}\left(\hat{\mathbf{q}}_{k}^{-}\right)\left[\mathbf{b}_{m} \times\right]}{\sqrt{\ell_{m}}}-\frac{\left(\mathbf{r}+A^{\top}\left(\hat{\mathbf{q}}_{k}^{-}\right) \mathbf{b}_{s_{i}}-\mathbf{t}_{j}\right)^{\top} A^{\top}\left(\hat{\mathbf{q}}_{k}^{-}\right)\left[\mathbf{b}_{s_{i}} \times\right]}{\sqrt{\ell_{s_{i}}}}\right\}
\end{aligned}
$$

The prediction of the covariance matrix is obtained by

$$
\dot{\mathbf{P}}=\mathbf{f}_{\boldsymbol{x}} \mathbf{P}+\mathbf{P f}_{\boldsymbol{x}}^{\top}+\mathbf{g}_{\boldsymbol{x}} \mathbf{Q g _ { x } ^ { \top }}
$$

Then, the continuous-discrete EKF update equations are summarized by

$$
\begin{gathered}
\Delta \hat{\boldsymbol{x}}_{k}^{+}=K_{k}\left[\tilde{\boldsymbol{y}}_{k}-\mathbf{h}_{k}\left(\hat{\mathbf{q}}_{k}^{-}\right)\right] \\
\mathbf{P}_{k}^{+}=\left[\mathbf{I}_{6 \times 6}-K_{k} \mathbf{H}_{k}\right] \mathbf{P}_{k}^{-} \\
\hat{\mathbf{q}}_{k}^{+}=\delta \hat{\mathbf{q}}_{k}^{+} \otimes \hat{\mathbf{q}}_{k}^{-} \\
\hat{\boldsymbol{\beta}}_{k}^{+}=\hat{\boldsymbol{\beta}}_{k}^{-}+\Delta \hat{\boldsymbol{\beta}}_{k}^{+}
\end{gathered}
$$

where the Kalman gain matrix is obtained by

$$
K_{k}=\mathbf{P}_{k}^{-} \mathbf{H}_{k}^{\top}\left[\mathbf{H}_{k} \mathbf{P}_{k}^{-} \mathbf{H}_{k}^{\top}+\mathbf{R}_{k}\right]^{-1}
$$

\section{Unscented Filter}

The EKF may fail to estimate the correct estimates because it uses a linearization of the nonlinear system. Therefore, errors in truncating the Taylor series to first order should be small. Also, a zero-mean Gaussian random process is assumed. Therefore, the mean and covariance used in EKF are propagated by using only the first-order truncated linearization of the nonlinear system. The third and higher order moments are thus all zero. Therefore, the EKF has an error in the covariance expression when the fourth-order moments of the Gaussian distribution (kurtosis) are not zero. A better mean and covariance expression can be obtained by using the Unscented Filter (UF) developed by Julier, Uhlmann and DurrantWhyte $[25,36-39]$. The UF uses the same structure as the EKF, however, the mean and covariance propagations are different. The main idea of UF is that with a fixed number of parameters it should be easier to approximate a Gaussian distribution than to approximate an arbitrary nonlinear function. Although the UF needs more computations than the EKF, it has several advantages; 1) the expected error is lower than the EKF and 2) the UF avoids 
the derivation of Jacobian matrices. In fact, the UF is accurate to third order for Gaussian inputs for all nonlinearities and at least to second order for non-Gaussian inputs [37].

In this section, an UF is developed using PL phase measurements. The quaternion is used as the attitude parameter because it is singularity free and the kinematics equation is bilinear. However, since quaternions are not independent parameters, the normalization constraint should be satisfied. The sigma points generated by using quaternions will violate this constraint. To solve this problem the modified Rodrigues parameter (MRP) [31] is used to generate the sigma points, which can be converted into quaternions. Although the MRPs have singularity at a $360^{\circ}$, the error MRPs related with the error quaternions should not have singularities in practice. Also, the exact form of quaternion propagation solution can still be used. This technique is first introduced by Crassidis and Markley [40] and called the unscented quaternion estimator (USQUE). Let the state vector be

$$
\hat{\boldsymbol{x}}_{k}^{+}=\left[\begin{array}{c}
\delta \hat{\mathbf{p}}_{k}^{+} \\
\hat{\boldsymbol{\beta}}_{k}^{+}
\end{array}\right]
$$

where $\delta \hat{\mathbf{p}}_{k}$ is the error MRP and $\hat{\boldsymbol{\beta}}_{k}$ is the bias error. For the application of the UF, a modified covariance matrix is given by [40]

$$
\mathbf{Q}_{k}=\frac{\Delta t}{2}\left[\begin{array}{cc}
\left(\sigma_{v}^{2}-\frac{1}{6} \sigma_{u}^{2} \Delta t^{2}\right) \mathbf{I}_{3 \times 3} & \mathbf{0}_{3 \times 3} \\
\mathbf{0}_{3 \times 3} & \sigma_{u}^{2} \mathbf{I}_{3 \times 3}
\end{array}\right]
$$

Then, the sigma points are generated by

$$
\begin{gathered}
\boldsymbol{\sigma}_{k} \leftarrow 2 n \text { columns from } \pm \sqrt{(n+\lambda) \mathbf{P}_{k}+\mathbf{Q}_{k}} \\
\chi_{k}(0)=\hat{\boldsymbol{x}}_{k} \\
\chi_{k}(i)=\boldsymbol{\sigma}_{k}(i)+\hat{\boldsymbol{x}}_{k}
\end{gathered}
$$

where $n$ is the dimension of the state and $\lambda=\left\{\alpha^{2}(n+\kappa)-n\right\}$ is a scaling parameter. The parameter $\kappa$ is a secondary scaling parameter which is usually set to 0 . The parameter $\alpha$ is usually set to a small positive value, and $\beta$ is used to compensate the higher-order moments. Setting $\beta=2$ is optimal for Gaussian distributions. Efficiently methods to compute the matrix square root can be found by using the Cholesky decomposition. If an orthogonal matrix square root is used, then the sigma points lie along the eigenvectors of the covariance matrix. Note that there are a total of $2 n$ values for $\boldsymbol{\sigma}_{k}$ (the positive and negative square roots). The mean and covariance of these points are known [25]. Since this set of points is symmetric, its odd central moments are zero, so its first three moments are the same as the original Gaussian distribution.

By using those sigma points the corresponding error quaternions can be generated by

$$
\begin{gathered}
\delta q_{4}^{+}(k, i)=\frac{1-\left\|\chi_{k}^{\delta \mathbf{p}}(i)\right\|^{2}}{1+\left\|\chi_{k}^{\delta \mathbf{p}}(i)\right\|^{2}} \\
\delta \mathbf{q}_{13}^{+}(k, i)=\left[1+\delta q_{4}^{+}(k, i)\right] \chi_{k}^{\delta \mathbf{p}}(i)
\end{gathered}
$$

where $\chi^{\delta \mathbf{p}}$ represents the sigma points pertaining to the error MRPs. Then, the propagation of the error quaternions are given by

$$
\begin{gathered}
\hat{\mathbf{q}}_{k+1}^{-}(i)=\Omega\left[\hat{\boldsymbol{\omega}}_{k}^{+}(i)\right] \hat{\mathbf{q}}_{k}^{+} \\
\delta \mathbf{q}_{k+1}^{-}(i)=\hat{\mathbf{q}}_{k+1}^{-}(i) \otimes\left[\hat{\mathbf{q}}_{k+1}^{-}(0)\right]^{-1}
\end{gathered}
$$

where

$$
\hat{\mathbf{q}}_{k}^{+}(0)=\hat{\mathbf{q}}_{k}^{+}, \quad \hat{\mathbf{q}}_{k}^{+}(i)=\delta \mathbf{q}_{k}^{+}(i) \otimes \hat{\mathbf{q}}_{k}^{+}
$$


Then, the propagated sigma points are given by

$$
\begin{gathered}
\chi_{k+1}(0) \equiv\left[\begin{array}{c}
\chi_{k+1}^{\delta \mathbf{p}}(0) \\
\chi_{k+1}^{\beta}(0)
\end{array}\right]=\left[\begin{array}{c}
\mathbf{0} \\
\chi_{k}^{\beta}(0)
\end{array}\right] \\
\chi_{k+1}(i) \equiv\left[\begin{array}{c}
\chi_{k+1}^{\delta \mathbf{p}}(i) \\
\chi_{k+1}^{\beta}(i)
\end{array}\right]=\left[\begin{array}{c}
\frac{\delta \mathbf{q}_{13}^{-}(k+1, i)}{1+\delta q_{4}^{-}(k+1, i)} \\
\chi_{k}^{\beta}(i)
\end{array}\right]
\end{gathered}
$$

The predicted mean for the state estimate is calculated by

$$
\hat{\boldsymbol{x}}_{k+1}^{-}=\frac{1}{n+\lambda}\left[\lambda \boldsymbol{\chi}_{k}(0)+\frac{1}{2} \sum_{i=1}^{2 n} \boldsymbol{\chi}_{k+1}(i)\right]
$$

The predicted covariance is given by

$$
\begin{gathered}
\mathbf{P}_{k+1}^{-}=\frac{1}{n+\lambda}\left\{\left[\lambda+\left(1-\alpha^{2}+\beta\right)(n+\lambda)\right]\left[\boldsymbol{\chi}_{k+1}(0)-\hat{\boldsymbol{x}}_{k+1}^{-}\right]\left[\boldsymbol{\chi}_{k+1}(0)-\hat{\boldsymbol{x}}_{k+1}^{-}\right]^{\top}\right. \\
\left.+\frac{1}{2} \sum_{i=1}^{2 n}\left[\boldsymbol{\chi}_{k+1}(i)-\hat{\boldsymbol{x}}_{k+1}^{-}\right]\left[\boldsymbol{\chi}_{k+1}(i)-\hat{\boldsymbol{x}}_{k+1}^{-}\right]^{\top}\right\}+\mathbf{Q}_{k}
\end{gathered}
$$

Since the observation $\gamma$ can be written as

$$
\gamma_{k+1}(i)=\left[\begin{array}{c}
\vdots \\
\frac{\left|\mathbf{r}+A^{\top}\left[\hat{\mathbf{q}}_{k+1}^{-}(i)\right] \mathbf{b}_{m}-\mathbf{t}_{j}\right|-\left|\mathbf{r}+A^{\top}\left[\hat{\mathbf{q}}_{k+1}^{-}(i)\right] \mathbf{b}_{s_{i}}-\mathbf{t}_{j}\right|}{\lambda}+n_{i j}+\tau_{i} \\
\vdots
\end{array}\right]
$$

The mean observation is obtained by

$$
\hat{\boldsymbol{y}}_{k+1}^{-}=\frac{1}{n+\lambda}\left[\lambda \boldsymbol{\gamma}_{k+1}(0)+\frac{1}{2} \sum_{i=1}^{2 n} \boldsymbol{\gamma}_{k+1}(i)\right]
$$

The output covariance is given by

$$
\begin{gathered}
\mathbf{P}_{k+1}^{y y}=\frac{1}{n+\lambda}\left\{\left[\lambda+\left(1-\alpha^{2}+\beta\right)(n+\lambda)\right]\left[\boldsymbol{\gamma}_{k+1}(0)-\hat{\boldsymbol{y}}_{k+1}^{-}\right]\left[\boldsymbol{\gamma}_{k+1}(0)-\hat{\boldsymbol{y}}_{k+1}^{-}\right]^{\top}\right. \\
\left.+\frac{1}{2} \sum_{i=1}^{2 n}\left[\boldsymbol{\gamma}_{k+1}(i)-\hat{\boldsymbol{y}}_{k+1}^{-}\right]\left[\boldsymbol{\gamma}_{k+1}(i)-\hat{\boldsymbol{y}}_{k+1}^{-}\right]^{\top}\right\}
\end{gathered}
$$

Then the innovations covariance is given by

$$
\mathbf{P}_{k+1}^{\nu \nu}=\mathbf{P}_{k+1}^{y y}+\mathbf{R}_{k+1}
$$

The cross covariance matrix is determined using

$$
\begin{gathered}
\mathbf{P}_{k+1}^{x y}=\frac{1}{n+\lambda}\left\{\left[\lambda+\left(1-\alpha^{2}+\beta\right)(n+\lambda)\right]\left[\boldsymbol{\chi}_{k+1}(0)-\hat{\boldsymbol{x}}_{k+1}^{-}\right]\left[\boldsymbol{\gamma}_{k+1}(0)-\hat{\boldsymbol{y}}_{k+1}^{-}\right]^{\top}\right. \\
\left.+\frac{1}{2} \sum_{i=1}^{2 n}\left[\boldsymbol{\chi}_{k+1}(i)-\hat{\boldsymbol{x}}_{k+1}^{-}\right]\left[\boldsymbol{\gamma}_{k+1}(i)-\hat{\boldsymbol{y}}_{k+1}^{-}\right]^{\top}\right\}
\end{gathered}
$$

The filter gain is then computed by

$$
\mathbf{K}_{k+1}=\mathbf{P}_{k+1}^{x y}\left(\mathbf{P}_{k+1}^{\nu \nu}\right)^{-1}
$$

The state vector can now be updated by

$$
\hat{\boldsymbol{x}}_{k+1}^{+}=\hat{\boldsymbol{x}}_{k+1}^{-}+\mathbf{K}_{k+1} \boldsymbol{\nu}_{k+1}
$$




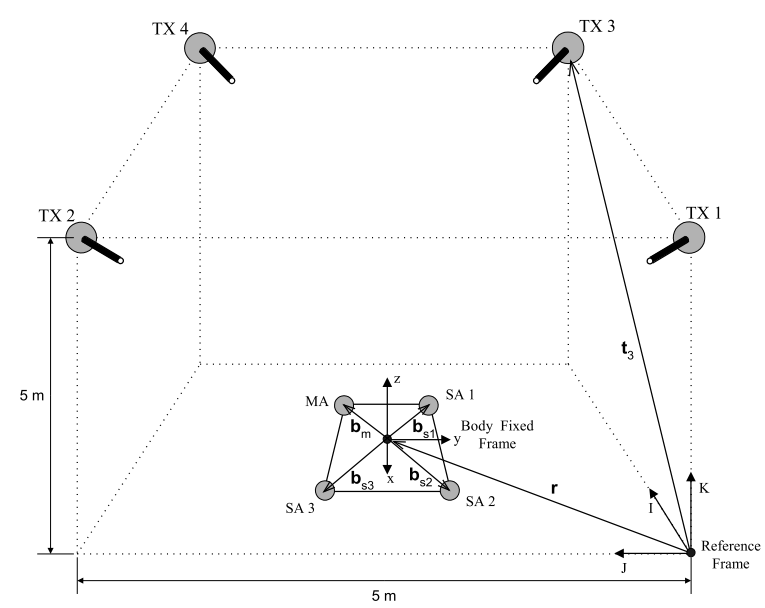

Figure 2: Geometric Configuration of Simulation

$$
\mathbf{P}_{k+1}^{+}=\mathbf{P}_{k+1}^{-}-\mathbf{K}_{k+1} \mathbf{P}_{k+1}^{\nu \nu} \mathbf{K}_{k+1}^{\top}
$$

The updates of MRPs and the biases are taken by using Eq. (50). Then, the quaternions are updated by

$$
\hat{\mathbf{q}}_{k+1}^{+}=\delta \hat{\mathbf{q}}_{k+1}^{+} \otimes \hat{\mathbf{q}}_{k+1}^{-}(0)
$$

where $\delta \hat{\mathbf{q}}_{k+1}^{+}$is given by

$$
\begin{gathered}
\delta q_{4}^{+}(k+1)=\frac{1-\left\|\delta \hat{\mathbf{p}}_{k+1}^{+}\right\|^{2}}{1+\left\|\delta \hat{\mathbf{p}}_{k+1}^{+}\right\|^{2}} \\
\delta \mathbf{q}_{13}^{+}(k+1)=\left[1+\delta q_{4}^{+}(k+1)\right] \delta \hat{\mathbf{p}}_{k+1}^{+}
\end{gathered}
$$

For the next step the states should be set as

$$
\hat{\boldsymbol{x}}_{k+1}^{+}=\left[\begin{array}{c}
\mathbf{0} \\
\Delta \boldsymbol{\beta}_{k+1}^{+}
\end{array}\right]
$$

The procedures of USQUE are as follows. First, the sigma points are generated by using some initial covariance. Then, they are transformed into error quaternions. The quaternions are propagated using Eq. (39) and the error quaternions are computed again. Then, the propagated sigma points and observations are obtained by using Eqs. (41) and (44). The predicted mean and covariance are then computed by using Eqs. (42), (45), (43), (46), and (48). Then, the update of covariance and error MRP are obtained by Eqs (50) and (49). Next, the update of the quaternion is accomplished by using the updated error MRP. Finally, the state is reset using Eq. (53) for the next propagation.

\section{Simulation and Results}

Pseudolite signals are simulated using the geometric configuration shown in Figure 2, The locations of the PL TXs and receiver antennas are displayed as TX1, TX2, TX3, TX4, MA, SA1, SA2, and SA3, respectively. The unit vectors $\{I, J, K\}$ are for the reference coordinate system and $\{x, y, z\}$ are for the body fixed coordinate system. The coordinates of the transceivers in the reference frame and those of the antennas in the body fixed frame are shown in Table 1. It is assumed that the position of the origin of the body fixed frame, $\mathbf{r}=[2.5,2.5,0]^{\mathrm{\top}}(\mathrm{m})$, is given from another source such as navigation data. Then, using these PL signal phase measurements, the characteristics of each attitude determination algorithm are investigated. 

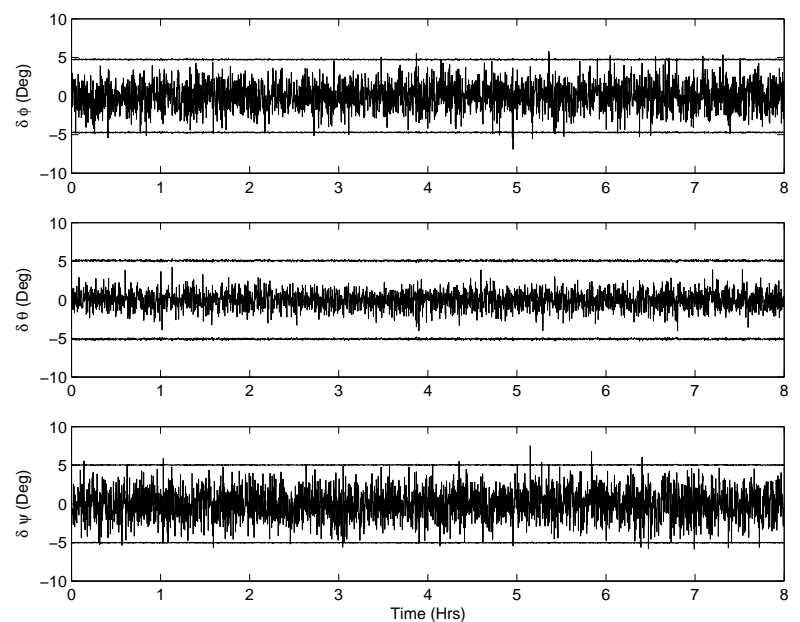

Figure 3: LM Method Euler Angle Errors (Static)

\section{LM Method}

Figure 3 shows the Euler angle errors and their 3- $\sigma$ boundary layers of the LM method. As can be seen, the errors are well inside $3-\sigma$ bounds. Also, we can notice that the errors in the pitch axis are smaller than those of the other axes when we use the sightline geometry shown in Figure 2 .

\section{Nonlinear Predictive Filter}

To investigate the behaviour of the Predictive filter, three different angular velocities are considered. First, the static case is considered to compare with the LM method. Then, cases of a LEO spacecraft having an orbital period of 95 minutes are considered. The angular velocities and weighting matrices of all cases are shown in Table 2 . Since both $\Delta t$ and $\mathbf{W}$ can affect the filter performance various values are chosen. The sampling interval, $\Delta t$, is set to 10 seconds.

\section{Static Case}

To show the convergence behavior large initial errors are considered:

$$
\left[\begin{array}{l}
\delta \phi \\
\delta \theta \\
\delta \psi
\end{array}\right]=\left[\begin{array}{l}
-43.086^{\circ} \\
-13.851^{\circ} \\
-55.382^{\circ}
\end{array}\right]
$$

A comparison of the Euler angle error is shown in Figure 4. The horizontal axis represents the time in hours unit and the longitudinal axis is the roll angle error in degrees. The $3-\sigma$ error bounds are also displayed in the figure. As can be seen, the roll angle error converges after 8 minutes for $\mathbf{W}=10^{6} \mathbf{I}$. However, the error clearly decreases as the weighting increases.

Table 1: Locations of Transceivers and Antennas

\begin{tabular}{|c|c|c|c|}
\hline Transceivers & Locations $(\mathrm{m})$ & Antennas & Locations $(\mathrm{m})$ \\
\hline \hline $\mathbf{t}_{1}$ & {$[0,0,5]^{\top}$} & $\mathbf{b}_{m}$ & {$[-0.05,-0.05,0]^{\top}$} \\
\hline $\mathbf{t}_{2}$ & {$[0,5,5]^{\top}$} & $\mathbf{b}_{s_{1}}$ & {$[0.05,-0.05,0]^{\top}$} \\
\hline $\mathbf{t}_{3}$ & {$[5,0,5]^{\top}$} & $\mathbf{b}_{s_{2}}$ & {$[0.05,0.05,0]^{\top}$} \\
\hline $\mathbf{t}_{4}$ & {$[5,5,5]^{\top}$} & $\mathbf{b}_{s_{3}}$ & {$[-0.05,0.05,0]^{\top}$} \\
\hline
\end{tabular}


Table 2: Angular Velocities and Weighting Matrices for Each Case

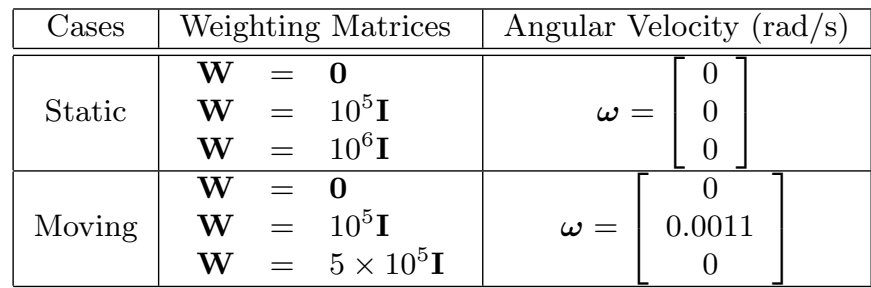
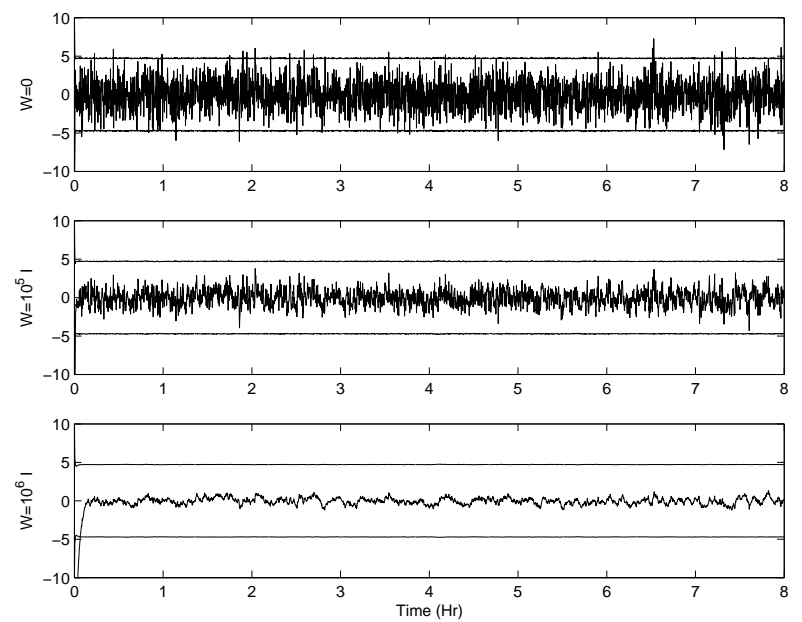

Figure 4: Attitude Error Comparison in the Roll Axis

To investigate the characteristics of the estimation errors, zero initial errors are considered with the weight matrices $\mathbf{W}=10^{5} \mathbf{I}, \mathbf{W}=10^{4} \mathbf{I}, \mathbf{W}=10^{3} \mathbf{I}$, and $\mathbf{W}=\mathbf{0}$. To investigate the statistical properties of the estimation errors, the Gaussian distribution of the roll angle errors are compared in Figure 5. As can be seen, the standard deviation decreases as the weighting increases. The mean and the standard deviation values are shown in Table 3 . For $\mathbf{W}=\mathbf{0}$, which is the deterministic case, the $3-\sigma$ values of Euler angle errors are over 6 degrees. However, for $\mathbf{W}=10^{5} \mathbf{I}$, the $3-\sigma$ values of Euler angle errors are less than 0.4 degrees. From the static simulation survey, we conclude that the larger weighting introduces smaller attitude errors. However, the convergence to the correct attitude is the slower.

\section{Moving Case}

To investigate the attitude determination performance of the Predictive filter in motion, a LEO orbit motion shown in Table 2 is considered. Pitch angle error is compared in Figure 6. At first as the weighting increases the Euler angle errors decrease. However, unlike the static case the errors seem to be biased for larger weightings. As a conclusion, for the static case the estimation error of the Predictive filter decreases as the weight increases. However,

Table 3: Mean and Standard Deviation Values

\begin{tabular}{|l|r|r|r|c|c|c|}
\hline & \multicolumn{3}{|c|}{ Mean (Deg.) } & \multicolumn{3}{c|}{ Standard Deviation (Deg.) } \\
\hline Weighting & Roll & Pitch & Yaw & Roll & Pitch & Yaw \\
\hline \hline $\mathbf{W}=10^{5} \mathbf{I}$ & 0.0755 & -0.1059 & 0.0341 & 0.1085 & 0.1061 & 0.1237 \\
\hline $\mathbf{W}=10^{4} \mathbf{I}$ & 0.0708 & -0.1248 & 0.0540 & 0.3787 & 0.3945 & 0.4120 \\
\hline $\mathbf{W}=10^{3} \mathbf{I}$ & 0.0690 & -0.1170 & 0.0499 & 1.0880 & 1.0511 & 0.9850 \\
\hline $\mathbf{W}=\mathbf{0}$ & 0.0428 & -0.1501 & 0.0891 & 2.0120 & 2.1646 & 1.9007 \\
\hline
\end{tabular}




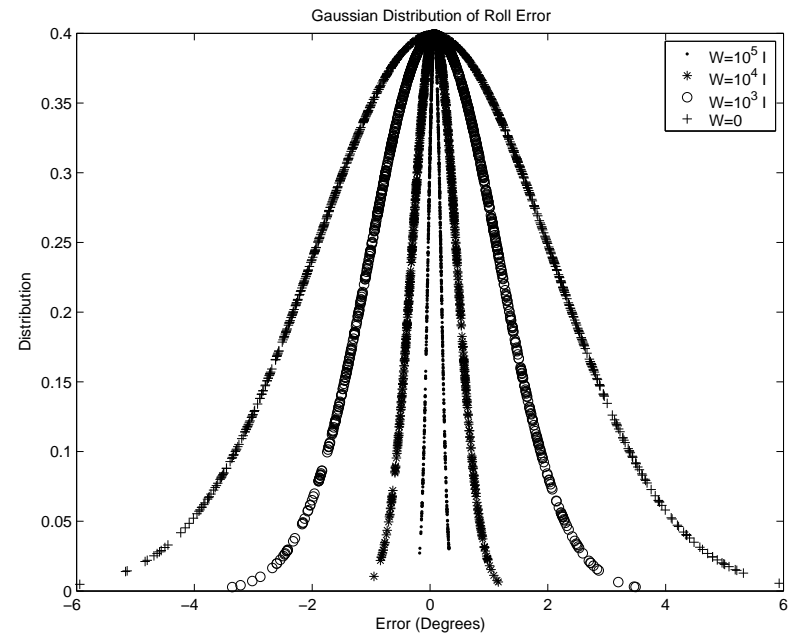

Figure 5: Attitude Error Distribution in the Roll Axis
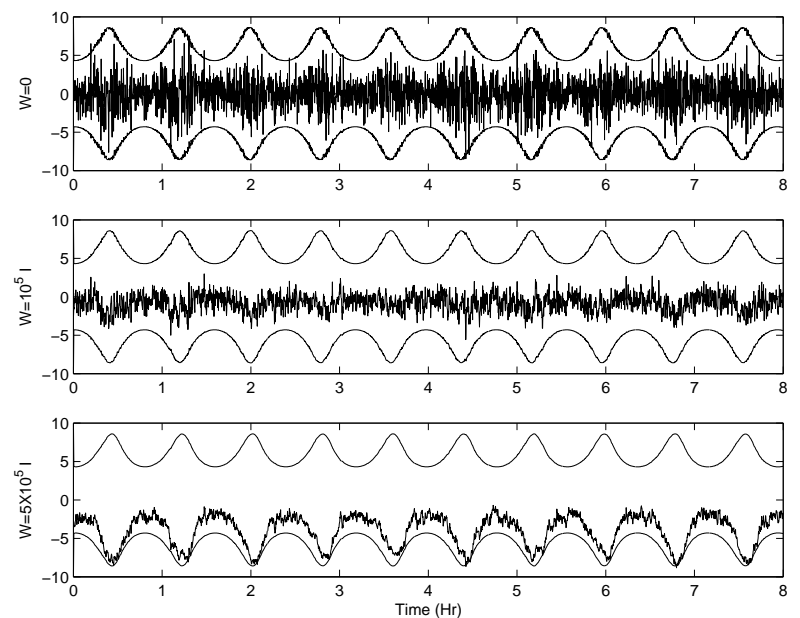

Figure 6: Attitude Error Comparison in the Pitch Axis (Moving-1) 

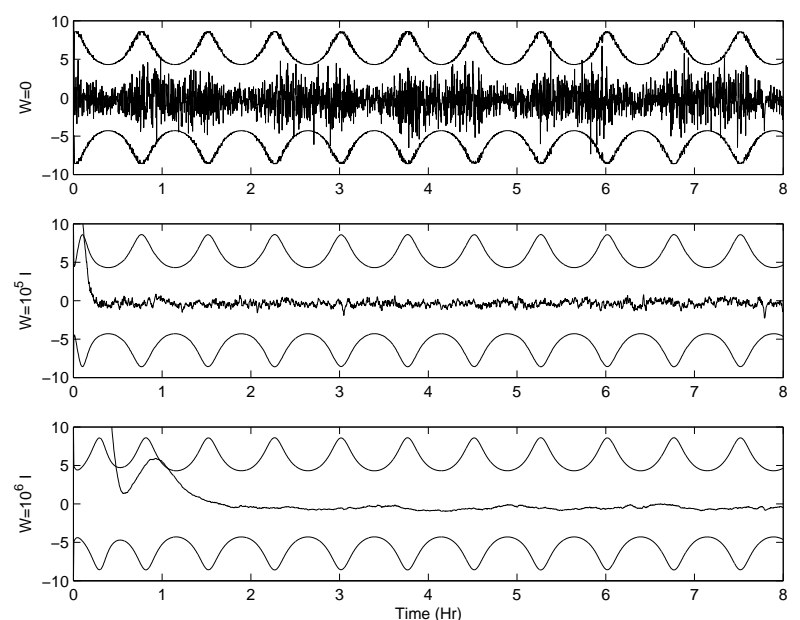

Figure 7: Attitude Error Comparison in the Pitch Axis

for the moving cases if the weights exceed certain limits the estimation errors increase and can violate the $3-\sigma$ bounds.

\section{Case using angular rate measurements}

When the angular rate measurements are available, we can still use the modified ALLEGRO by using model given by

$$
\dot{\hat{\mathbf{q}}}=\frac{1}{2} \boldsymbol{\Xi}(\hat{\mathbf{q}})[\tilde{\boldsymbol{\omega}}+\mathbf{d}]
$$

Then, we have new form of $\boldsymbol{z}$ variable as

$$
\boldsymbol{z}=\frac{1}{2 \lambda}\left[\begin{array}{c}
\frac{\hat{\mathbf{q}}^{\top}\left[\boldsymbol{\Omega}\left(\mathbf{b}_{s_{1}}\right) \boldsymbol{\Gamma}\left(\mathbf{t}_{1}-\mathbf{r}\right)+\boldsymbol{\Gamma}\left(\mathbf{t}_{1}-\mathbf{r}\right) \boldsymbol{\Omega}\left(\mathbf{b}_{s_{1}}\right)\right]}{\sqrt{\ell+\mathbf{b}_{s_{1}}^{\top} \mathbf{b}_{s_{1}}-2 \hat{\mathbf{q}}^{\top} \boldsymbol{\Omega}\left(\mathbf{b}_{s_{1}}\right) \boldsymbol{\Gamma}\left(\mathbf{t}_{1}-\mathbf{r}\right) \hat{\mathbf{q}}}}-\frac{\hat{\mathbf{q}}^{\top}\left[\boldsymbol{\Omega}\left(\mathbf{b}_{m}\right) \boldsymbol{\Gamma}\left(\mathbf{t}_{1}-\mathbf{r}\right)+\boldsymbol{\Gamma}\left(\mathbf{t}_{1}-\mathbf{r}\right) \boldsymbol{\Omega}\left(\mathbf{b}_{m}\right)\right]}{\sqrt{\ell+\mathbf{b}_{m}^{\top} \mathbf{b}_{m}-2 \hat{\mathbf{q}}^{\top} \boldsymbol{\Omega}\left(\mathbf{b}_{m}\right) \boldsymbol{\Gamma}\left(\mathbf{t}_{1}-\mathbf{r}\right) \hat{\mathbf{q}}}} \\
\vdots \\
\frac{\hat{\mathbf{q}}^{\top}\left[\boldsymbol{\Omega}\left(\mathbf{b}_{s_{m}}\right) \boldsymbol{\Gamma}\left(\mathbf{t}_{n}-\mathbf{r}\right)+\boldsymbol{\Gamma}\left(\mathbf{t}_{n}-\mathbf{r}\right) \boldsymbol{\Omega}\left(\mathbf{b}_{s_{m}}\right)\right]}{\sqrt{\ell+\mathbf{b}_{s_{m}}^{\top} \mathbf{b}_{s_{m}}-2 \hat{\mathbf{q}}^{\top} \boldsymbol{\Omega}\left(\mathbf{b}_{s_{m}}\right) \boldsymbol{\Gamma}\left(\mathbf{t}_{n}-\mathbf{r}\right) \hat{\mathbf{q}}}}-\frac{\hat{\mathbf{q}}^{\top}\left[\boldsymbol{\Omega}\left(\mathbf{b}_{m}\right) \boldsymbol{\Gamma}\left(\mathbf{t}_{n}-\mathbf{r}\right)+\boldsymbol{\Gamma}\left(\mathbf{t}_{n}-\mathbf{r}\right) \boldsymbol{\Omega}\left(\mathbf{b}_{m}\right)\right]}{\sqrt{\ell+\mathbf{b}_{m}^{\top} \mathbf{b}_{m}-2 \hat{\mathbf{q}}^{\top} \boldsymbol{\Omega}\left(\mathbf{b}_{m}\right) \boldsymbol{\Gamma}\left(\mathbf{t}_{n}-\mathbf{r}\right) \hat{\mathbf{q}}}}
\end{array}\right] \boldsymbol{\Xi}(\hat{\mathbf{q}}) \tilde{\boldsymbol{\omega}}
$$

Figure 7 shows the pitch angle estimation errors for three different weightings. The angular rate measurements error characteristics are the same as those used in EKF and UF comparison. Also, to investigate the large initial errors case, the same large initial Euler angle errors used in EKF and UF are applied, too. As can be seen, the pitch angle errors decrease when the weighting increases. However, its convergence is slow.

\section{EKF and UF}

The Pseudolite signal measurements as well as angular velocity measurements with gyros are used for the comparison of EKF and UF. The comparison is accomplished in two parts. The first compares the mean estimation error through Monte Carlo like simulations. By using this comparison, the statistical properties of the EKF and UF can be investigated. Then, by using large initial errors the convergence behaviors are investigated. 


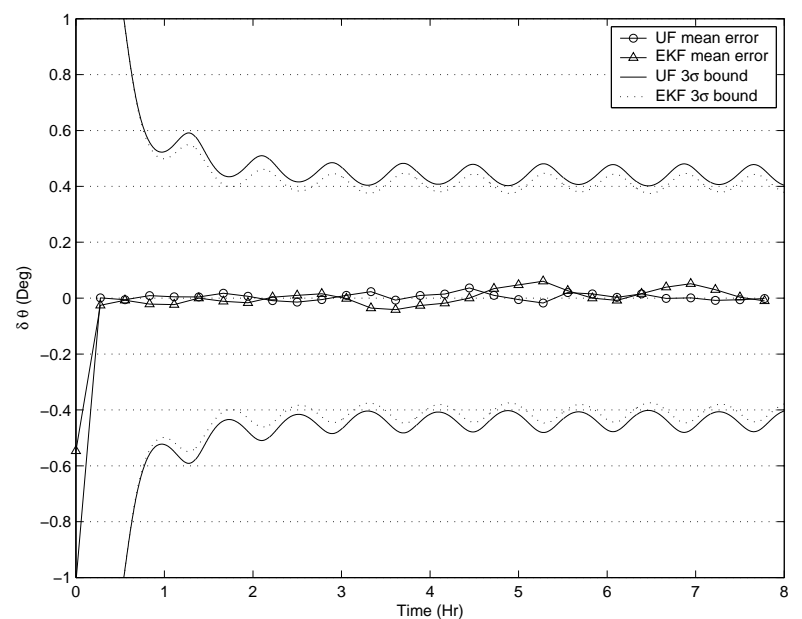

Figure 8: EKF vs. UF Pitch Error

\section{Monte Carlo Simulation}

To compare the EKF and UF, a Monte Carlo simulation is applied. The initial Euler angle errors and gyro drift errors are generated randomly by

$$
\begin{aligned}
{\left[\begin{array}{l}
\delta \phi \\
\delta \theta \\
\delta \psi
\end{array}\right] } & =30 \operatorname{randn}(3,1)(\mathrm{Deg}) \\
\Delta \boldsymbol{\beta} & =.0001 \operatorname{randn}(3,1)(\mathrm{rad} / \mathrm{sec})
\end{aligned}
$$

where the 'randn' is the MATLAB command used to generate a Gaussian normal random number whose standard deviation is 1 . The initial covariance matrix is set to

$$
\mathbf{P}_{0}=\left[\begin{array}{cc}
0.5 \mathbf{I}_{3 \times 3} & \mathbf{0}_{3 \times 3} \\
\mathbf{0}_{3 \times 3} & 10^{-5} \mathbf{I}_{3 \times 3}
\end{array}\right]
$$

The process covariance matrix is given by Eq. (36) where $\Delta t=10$ seconds, $\sigma_{v}=1.7222 \mathrm{e}-5$ $\left(\mathrm{rad} / \mathrm{sec}^{3 / 2}\right)$, and $\sigma_{u}=1.8133 \mathrm{e}-8\left(\mathrm{rad} / \mathrm{sec}^{3 / 2}\right)$. The measurement covariance matrix is given by $\mathbf{R}=\sigma_{p}^{2} \mathbf{I}_{12 \times 12}$ where $\sigma_{p}=0.0263$ (cycles).

By using 100 random initial attitude errors, bias errors, and measurements, the mean values of Euler angle errors and biases errors are compared in the following figures. Figure 8 shows the pitch angle estimation error comparison. As can be seen, the $3-\sigma$ bounds of UF are slightly larger than those of the EKF. However, this does not mean that the EKF's covariance is the smaller. Since the EKF uses a linearization approximation, the mean and covariance expression do have errors [36]. In actuality, the covariance expression of UF is more correct than the EKF. In fact, the differences in the mean estimation errors cannot be distinguishable. Also, the differences of $3-\sigma$ bounds between the UF and EKF are the smallest for the pitch axis while the values of 3- $\sigma$ bounds are the largest due to the sightline geometry. Figure 9 shows the gyro drift errors comparison. Now, the 3- $\sigma$ bounds of the UF are slightly smaller than those of the EKF. For the same reason in the Euler angle errors, the covariance of the UF is more reliable than that of the EKF. Similar to the Euler angles error, the differences in the mean value of the gyro drift error are not distinguishable while the differences in the 3- $\sigma$ bounds are slightly different. 


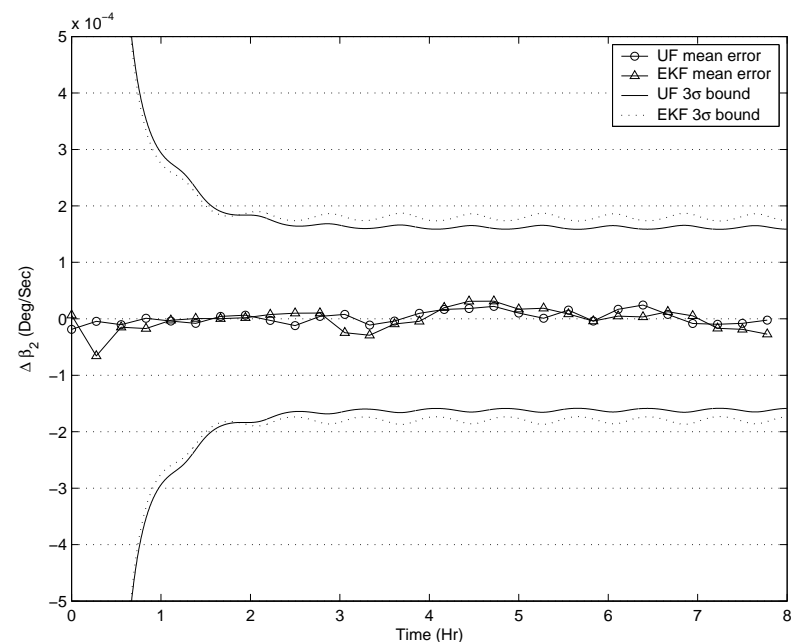

Figure 9: EKF vs. UF $\beta_{2}$ Error
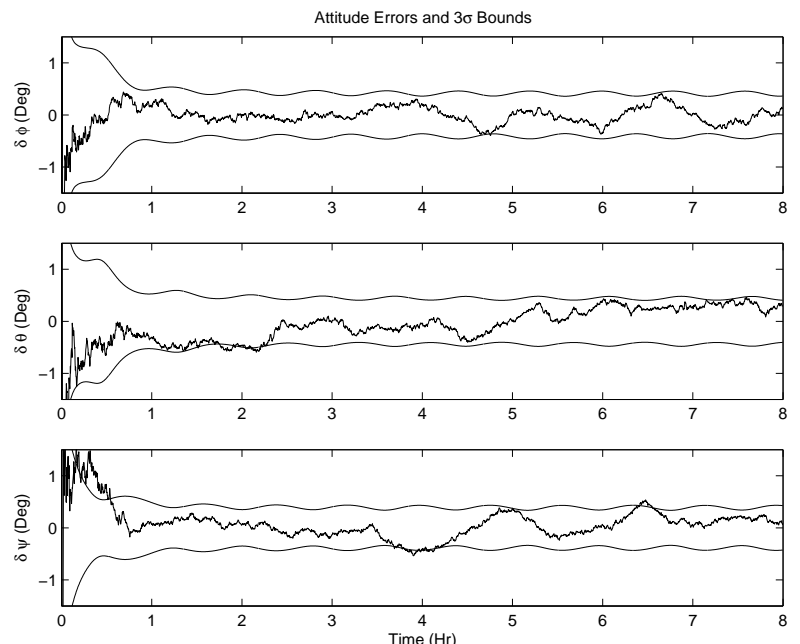

Figure 10: UF Attitude Estimation Errors

\section{Large Initial Errors}

To compare the convergence behavior of the EKF and UF, a large initial error is considered as

$$
\left[\begin{array}{l}
\delta \phi \\
\delta \theta \\
\delta \psi
\end{array}\right]=\left[\begin{array}{r}
0 \\
-120^{\circ} \\
0
\end{array}\right], \quad \Delta \boldsymbol{\beta}=\left[\begin{array}{r}
-0.0001 \\
0.0003 \\
0.0002
\end{array}\right] \quad(\mathrm{rad} / \mathrm{sec})
$$

With the given initial guesses, the Euler angle estimation errors are compared in Figures 10 and 11. As can be seen, the estimation of the EKF fails while the UF estimation errors are well within their 3- $\sigma$ bounds, although the convergence of the covariance and estimation error requires an hour. The failure of the EKF is caused by the linearization approximation of the EKF that works only for small, first-order, errors. Similar to Euler angle errors, the gyro drift estimation of the EKF fails. However, the estimation errors of the UF seem to be within 3- $\sigma$ bounds. Also, the convergence of the drift errors requires more time than Euler angle estimation. 

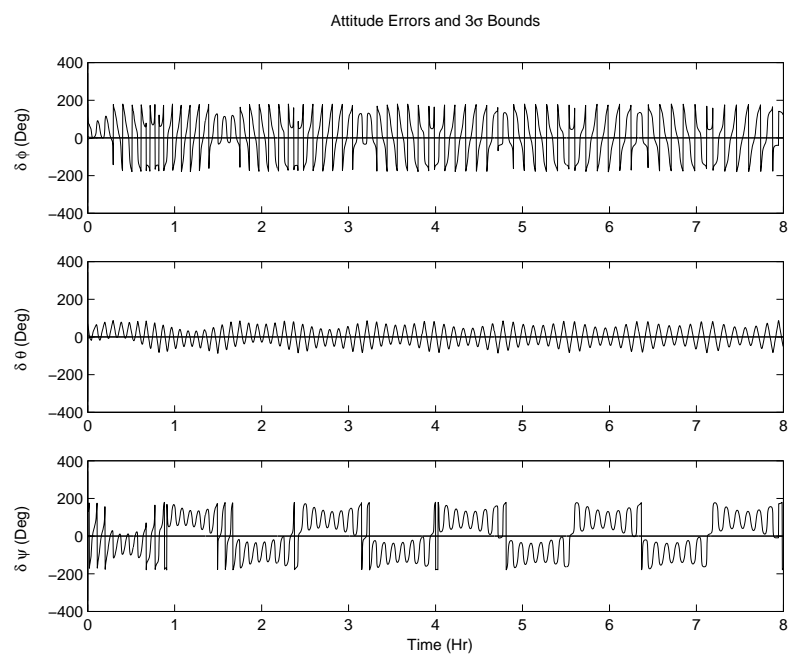

Figure 11: EKF Attitude Estimation Errors

\section{Concluding Remarks}

Attitude estimation algorithms using pseudolite signals were developed and compared. The comparison is separated into two cases, the static and the moving cases. For the static case, the Levenberg-Marquardt algorithm is compared with the modified ALLEGRO. As a result, the modified ALLEGRO with large weighting shows the smallest estimation errors while the estimation error covariance of all methods satisfies the Cramér-Rao lower bounds. For the moving case, we consider a LEO spacecraft motion. Since the iterative algorithm, LM, is not efficient, only the modified ALLEGRO, EKF, and UF are compared. The estimation errors of the nonlinear predictive filter decrease as the weighting increases. However, unlike the static case, the estimation error of the modified ALLEGRO increases as the weight increases after a certain value of weighting which is varying with respect to sampling interval, $\Delta t$, and spacecraft angular motion. This problem can be solved by using angular rate measurements.

In the comparison of large initial errors behavior, the modified ALLEGRO and UF provide more robust results than EKF. However, both the EKF and UF determine the attitude well within 0.5 degrees Euler angle errors while the modified ALLEGRO seems to have smaller residual errors. So the best choice is to use the modified ALLEGRO in the beginning then switch into either the EKF or UF using those estimates as the initial guesses.

\section{Acknowledgement}

This work was supported under a NASA grant (NASA \#5-7510), under the supervision of Ms. Janet Bell. The authors greatly appreciate this support as well as help from the Navigation Systems and Technology Laboratory at NASA - Johnson Space Center.

\section{References}

[1] Cohen, C., Attitude Determination Using GPS, Ph.D. dissertation, Stanford University, Dec. 1992.

[2] Crassidis, J., Markley, F., Lightsey, E., and Ketchum, E., "Predictive Attitude Estimation Using Global Positioning System Signals," Proceedings of the Flight Mechanics/Estimation Theory Symposium, NASA-Goddard Space Flight Center, Greenbelt, MD, May 1997, pp. 107-120. 
[3] Crassidis, J., Lightsey, E., and Markley, F., "Efficient and Optimal Attitude Determination Using Recursive Global Positioning System Signal Operations," AIAA Journal of Guidance, Control, and Dynamics, Vol. 22, No. 2, March-April 1999, pp. 193-201.

[4] Park, K. and Crassidis, J., "Autonomous Attitude Determination for ISS Applications Using Pseudolite Signals," Tech. Rep. , NASA Johnson Space Center, Houston, TX, June 2003.

[5] Gaylor, D. and Lightsey, E., "GPS/INS Kalman Filter Design for Spacecraft Operating in the Proximity of the International Space Station," [CD-ROM] AIAA Guidance, Navigation, and Control Conference and Exhibit, Austin, TX, Aug. 2003.

[6] Crassidis, J., Markley, F., and Lightsey, E., "Global Positioning System Integer Ambiguity Resolution Without Attitude Knowledge," AIAA Journal of Guidance, Control, and Dynamics, Vol. 22, No. 2, March-April 1999, pp. 212-218.

[7] Lightsey, E. and Crassidis, J., "Real Time Attitude Independent GPS Integer Ambiguity Resolution," The John L. Junkins Astrodynamics Symposium, College Station, TX, May 2003, pp. 145-164.

[8] Lightsey, E., Crassidis, J., and Markley, F., "Fast Integer Ambiguity Resolution for GPS Attitude Determination," Proceedings of the AIAA Guidance, Navigation, and Control Conference, Portland, OR, Aug. 1999, pp. 403-412.

[9] Comp, C. and Axelrad, P., "Adaptive SNR-Based Carrier Phase Multipath Mitigation Technique," IEEE Transactions on Aerospace and Electric Systems, Vol. 34, No. 1, Jan. 1998, pp. 264-276.

[10] Wahba, G., "A Least Squares Estimate of Spacecraft Attitude," SIAM Review, Vol. 7, No. 3, July 1965, pp. 409.

[11] Bar-Itzhack, I., Montgomery, P., and Garrick, J., "Algorithms for Attitude Determination Using GPS," Proceedings of the AIAA Guidance, Navigation, and Control Conference, Reston, VA, Aug. 1997, pp. 841-851.

[12] Crassidis, J. and Markley, F., "A New Algorithm for Attitude Determination Using Global Positioning System Signals," Journal of Guidance, Control, and Dynamics, Vol. 20, No. 5, Sept.-Oct. 1997, pp. 891-896.

[13] Crassidis, J., Markley, F., and Lightsey, E., "Application of Vectorized Attitude Determination Using Global Positioning System Signals," Proceedings of the AIAA/AAS Astrodynamics Specialist Conference, Boston, MA, Aug. 1998.

[14] Crassidis, J. and Markley, F., "A Predictive Attitude Determination Algorithm," Proceedings of the Flight Mechanics/Estimation Theory Symposium, NASA-Goddard Space Flight Center, Greenbelt, MD, May 1997, pp. 249-263.

[15] Fujikawa, S. and Zimbelman, D., "Spacecraft Attitude Determination by Kalman Filtering of Global Positioning System Signal," Journal of Guidance Control and Dynamics, Vol. 18, No. 6, Nov.-Dec. 1995, pp. 1365-1371.

[16] Axelrad, P. and Behre, C., "Satellite Attitude Determination Based on GPS Signal-toNoise Ratio," Proceedings of the IEEE, Vol. 87, No. 1, Jan. 1999, pp. 133-144.

[17] Madsen, J. and Lightsey, E., "Attitude Determination Using GPS Signal to Noise Ratio and Carrier Phase Measurements," [CD-ROM] AIAA Guidance, Navigation, and Control Conference and Exhibit, Austin, TX, Aug. 2003.

[18] Melvin, P. and Hope, A., "Satellite Attitude Determination with GPS," Advances in the Astronautical Sciences, Vol. 85 - Part I, 1993, pp. 59-78. 
[19] Melvin, P., Ward, L., and Axelrad, P., "The Analysis of GPS Attitude Data from a Slowly Rotating, Symmetrical Gravity Gradient Satellite," Advances in the Astronautical Sciences, Vol. 89 - Part I, 1995, pp. 539-558.

[20] Lightsey, E., Cohen, C., Feess, W., and Parkinson, B., "Analysis of Spacecraft Attitude Measurements Using Onboard GPS," 17th Annual American Astronautical Society (AAS) Guidance and Control Conference, Feb. 1994, pp. 521-532.

[21] Lightsey, E., Ketchum, E., Flatley, T., Crassidis, J., Freesland, D., Reiss, K., and Young, D., "Flight Results of GPS-Based Attitude Control on the REX-II Spacecraft," Proceedings of ION GPS-96, Kansas City, MO, Sept. 1996, pp. 1037-1046.

[22] Brock, J., Fuller, R., Kemper, B., Mleczko, D., Rodden, J., and Tadros, A., "GPS Attitude Determination and Navigation Flight Experiment," Proceedings of ION GPS95, Palm Springs, CA, Sept. 1995, pp. 545-554.

[23] Zimmerman, K. and Cannon Jr., R., "Differential Carrier Phase GPS Techniques for Space Vehicle Rendezvous," Proceedings of ION GPS-94, Salt Lake City, UT, Sept. 1994, pp. 1693-1700.

[24] Zimmerman, K. and Cannon Jr., R., "Experimental Demonstration of GPS for Rendezvous Between Two Prototype Space Vehicles," Proceedings of ION GPS-95, Palm Springs, CA, Sept. 1995, pp. 1905-1913.

[25] Julier, S., Uhlmann, J., and Durrant-Whyte, H., "A New Method for the Nonlinear Transformation of Means and Covariances in Filters and Estimators," IEEE Transactions on Automatic Control, Vol. 45, No. 3, March 2000, pp. 477-482.

[26] Parkinson, B. and Spilker Jr., J., ed., Global Positioning System: Theory and Applications, Vol. I-II, American Institute of Aeronautics and Astronautics, Washington, DC, 1996.

[27] Hofmann-Wellenhof, B., Lichtenegger, H., and Collins, J., Global Positioning System: Theory and Practice, 4th ed., Springer-Verlag, New York, 1997.

[28] Dai, L., Wang, J., Tsujii, T., and Rizos, C., "Pseudolite Application in Positioning and Navigation: Modeling and Geometric Analysis," Int'l Symposium on Kinematic Systems in Geodesy, Geomatics \& Navigation (KIS2001), Banff, Canada, June 2001, pp. $482-489$.

[29] Stone, J., LeMaster, E., Powell, J., and Rock, S., "GPS Pseudolite Transceivers and Their Applications," Proceedings of National Technical Meeting 99, San Diego, CA, Jan. 1999, pp. 415-424.

[30] Teague, E., How, J., Lawson, L., and Parkinson, B., "Carrier Differential GPS for Real-Time Control of Large Flexible Structures," Proceedings of ION GPS-96, Kansas City, MO, Sept. 1996, pp. 1355-1365.

[31] Shuster, M., "A Survey of Attitude Representations," Journal of Astronautical Sciences, Vol. 41, No. 4, Oct. 1993, pp. 439-517.

[32] Bryson, Jr., A. and Ho, Y., Applied Optimal Control (Revised Printing), Taylor \& Francis, Levittown, PA, 1975.

[33] Crassidis, J. and Junkins, J., Optimal Estimation of Dynamic Systems, Chapman \& Hall/CRC, Boca Raton, FL, 2004.

[34] Crassidis, J. and Markley, F., "Predictive Filtering for Nonlinear Systems," Journal of Guidance, Control, and Dynamics, Vol. 20, No. 3, May-June 1997, pp. 566-572. 
[35] Lefferts, E., Markley, F., and Shuster, M., "Kalman Filtering for Spacecraft Attitude Estimation," Journal of Guidance, Control, and Dynamics, Vol. 5, No. 5, Sept. 1982, pp. 417-429.

[36] Julier, S. and Uhlmann, J., "A General Method for Approximating Nonlinear Transformations of Probability Distributions," Online, 1996, http://citeseer.ist.psu.edu/julier96general.html.

[37] van der Merwe, R. and Wan, E., "Sigma-Point Kalman Filters for Probabilistic Inference in Dynamic State-Space Models," Proceedings of the Workshop on Advances in Machine Learning, Montreal, Canada, June 2003, http://www.iro.umontreal.ca/ kegl/CRMWorkshop/paperMerweWan.pdf.

[38] van der Merwe, R., de Freitas, N., Doucet, A., and Wan, E., "The Unscented Particle Filter," Tech. Rep. CUED/F-INFENG/TR380, Cambridge University Engineering Department, Aug. 2000.

[39] van der Merwe, R. and Wan, E. A., "The Square-Root Unscented Kalman Filter For State And Parameter-Estimation," Online, http://citeseer.ist.psu.edu/ vandermerwe01squareroot.html.

[40] Crassidis, J. and Markley, F., "Unscented Filtering for Spacecraft Attitude Estimation," Journal of Guidance, Control, and Dynamics, Vol. 26, No. 4, July-Aug. 2003, pp. 536542. 